\title{
Sirtuins in Cancer: a Balancing Act between Genome Stability and Metabolism
}

\author{
Seung Min Jeong ${ }^{1,2, *}$, and Marcia C. Haigis ${ }^{3, *}$
}

\begin{abstract}
Genomic instability and altered metabolism are key features of most cancers. Recent studies suggest that metabolic reprogramming is part of a systematic response to cellular DNA damage. Thus, defining the molecules that fine-tune metabolism in response to DNA damage will enhance our understanding of molecular mechanisms of tumorigenesis and have profound implications for the development of strategies for cancer therapy. Sirtuins have been established as critical regulators in cellular homeostasis and physiology. Here, we review the emerging data revealing a pivotal function of sirtuins in genome maintenance and cell metabolism, and highlight current advances about the phenotypic consequences of defects in these critical regulators in tumorigenesis. While many questions should be addressed about the regulation and context-dependent functions of sirtuins, it appears clear that sirtuins may provide a promising, exciting new avenue for cancer therapy.
\end{abstract}

\section{INTRODUCTION}

Genomic instability is associated with most cancers and can be a marker of malignant transformation (Abbas and Dutta, 2009; Deng, 2006; Negrini et al., 2010). During tumorigenesis, genomic instability appears to facilitate the acquisition of tumorigenic mutations to support tumor cell survival and proliferation. Genomic instability can occur by environmental agents or normal cellular processes. For example, ionizing radiation (IR) and ultra-violet (UV), which can produce DNA lesions such as pyrimidine dimers, single-strand DNA breaks (SSBs) and doublestrand (DSBs), are well known genotoxic agents. Spontaneous DNA alterations can occur during normal DNA replication. Additionally, reactive oxygen species (ROS) produced by cellular metabolism can also induce DNA lesions.

\footnotetext{
${ }^{1}$ Department of Biochemistry, ${ }^{2}$ Institute for Aging and Metabolic Diseases College of Medicine, The Catholic University of Korea, Seoul 137-701, Korea, ${ }^{3}$ Department of Cell Biology, Harvard Medical School, Boston, MA, USA

*Correspondence: smjeong@catholic.ac.kr (SMJ); marcia haigis@hms. harvard.edu (MCH)
}

Received 12 June, 2015; revised 29 August, 2015; accepted 31 August, 2015; published online 18 September, 2015

Keywords: cancer, genomic stability, metabolism, sirtuins
To maintain genomic integrity, eukaryotic cells have developed a tightly coordinated DNA damage response (DDR) pathway. Upon DNA damage, ataxia telangiectasia mutated (ATM) and ataxia telangiectasia and RAD3-related protein (ATR) are activated and induce phosphorylation of downstream mediator proteins to trigger cell cycle arrest and DNA repair (Abraham, 2001; Ciccia and Elledge, 2010; Su, 2006) Indeed, dysregulations of DDR are commonly in associated with tumorigenesis and are observed in many tumors (Ciccia and Elledge, 2010; Negrini et al., 2010).

Tumors exhibit aberrant cellular metabolism characterized by elevated aerobic glycolysis, glutaminolysis and lipid metabolism. The Warburg effect, which describes that tumor cells preferentially use glucose for aerobic glycolysis in the presence of ample oxygen (Warburg, 1956), has emerged as a central metabolic hallmark of many cancers and indicates that alterations in metabolism contributes to the malignant phenotype (Tennant et al., 2010; Vander Heiden et al., 2009). In addition to glucose, many cancer cells exhibits enhanced glutamine metabolism and cannot survive in the absence of glutamine (Wise and Thompson, 2010). Recent studies have shown that a number of well-established oncogenic cues, including Myc, Ras or mTORC pathways play critical roles in inducing glutaminolysis (Csibi et al., 2013; Dang, 2010; Wang et al., 2010). Also, there is increasing evidence that tumor cells show alterations in lipid metabolism (Santos and Schulze, 2012). Changes in lipid metabolism contribute to membrane synthesis, energy homeostasis and cellular signaling. This metabolic reprogramming allows tumor cells to fulfill their bioenergetic and biosynthetic needs for rapid proliferation, and also provide additional metabolic advantages for their survival and growth under limited nutrient conditions (Gatenby and Gillies, 2004; Tong et al., 2009; Wise and Thompson, 2010).

Genomic instability and altered metabolism are hallmarks of many cancers. Thus, defining the connection between DDR and metabolic processes is an area of intense interest. Recent studies have shown that DNA damage also induces metabolic changes in cells (Cosentino et al., 2011), and many metabolic proteins, involved in glycolysis and glutaminolysis, are important component of cell cycle regulation and DNA repair (Ciccia and Elledge, 2010; Lapenna and Giordano, 2009). However, the molecular events and specific factors that are involved in regulating genomic stability and cell metabolism are not well elucidated.

Sirtuins are the homologs of the yeast Sir2 gene, which has been known to promote replicative life span and mediate gene silencing in yeast (Guarente and Kenyon, 2000; Haigis 
and Sinclair, 2010). Sirtuins function as nicotinamide adenine dinucleotide $\left(\mathrm{NAD}^{+}\right)$-dependent deacetylases, mono ADPribosyltransferases, lipoamidase (SIRT4), and deacylases (Du et al., 2011; Haigis and Sinclair, 2010; Mathias et al., 2014). Based on their enzymatic activities, sirtuins have been involved in a wide range of biological processes such as stress response, differentiation, proliferation, metabolism and aging. Due to their pivotal roles as regulators of cellular homeostasis in response to the cellular environment, mounting studies have investigated the roles of sirtuins in cancer (Haigis and Sinclair, 2010). This review provides an update on the functions of sirtuins in genome stability and metabolism, and further focusing on the implication of sirtuins in the interplay between stress response and metabolism.

\section{SIRTUINS IN GENOMIC STABILITY}

DNA damage initiates a tightly coordinated signaling response to maintain genomic integrity, and defects in the DDR often lead to increased incorporation of mutations into newly synthesized DNA, the accumulation of chromosomal instability and tumor development (Abbas and Dutta, 2009; Negrini et al., 2010). The DDR is primarily initiated by activation of related kinases ATM, ATR, and DNA-dependent protein kinase (DNA-PK) and by members of the poly (ADP-ribose) polymerase (PARP) family (Ciccia and Elledge, 2010). Following the recognition of DNA lesions, these sensor proteins induce phosphorylation of CHK1, CHK2 and $\gamma-\mathrm{H} 2 \mathrm{AX}$ to trigger cell cycle arrest and to initiate assembly of DNA damage repair machinery (Abraham, 2001; Ciccia and Elledge, 2010). Accruing evidence indicates that sirtuins have evolved to coordinate both stress response and cellular adaptation against genotoxic stress.

\section{In the nucleus and cytoplasm}

Mammals have seven sirtuins (SIRT1-7), three of which (SIRT1, 6 and 7) are mainly localized in the nucleus. SIRT1 is one of the best-characterized sirtuins. SIRT1 can remove the acetyl group from the lysine residues in histones and non-histone proteins (Finkel et al., 2009). In the past decade, numerous targets of SIRT1 have been identified, suggesting that SIRT1 plays a central role in a large variety of cellular processes. The involvement of SIRT1 in genomic stability has been supported by several lines of evidence. In response to DNA damage, SIRT1 is recruited to DNA DSBs in an ATM kinase-dependent manner (Oberdoerffer et al., 2008; Wang et al., 2008). This recruitment is important for $\gamma-\mathrm{H} 2 \mathrm{AX}$ foci formation and accumulation of the DDR-related proteins such as Rad51, NBS1 and BRCA1 at the breaks. Thus, Sirt1-deficient mouse embryonic fibroblasts (MEFs) exhibited a marked reduction of recruitment of these proteins after DNA damage (Oberdoerffer et al., 2008). In mouse embryos, absence of SIRT1 results in a large amount of chromosome abnormalities, and SIRT1 heterozygous deletion accelerates tumorigenesis in $\mathrm{p} 53^{+/-}$mice (Wang et al., 2008).

At the same time, SIRT1 is implicated in DNA repair pathways. When DNA DSBs occur, SIRT1 promotes homologous recombination (HR) DNA repair by deacetylating WRN, a member of the RecQ DNA helicase family with functions in maintenance of genomic stability (Li et al., 2008). Another studies have reported that SIRT1 interacts with telomere in vivo and SIRT1 overexpressed mice display increased HR DNA repair throughout the entire genome (Palacios et al., 2010). Moreover, SIRT1 is also involved in non-homologous end joining (NHEJ) DNA repair. Deacetylation of Ku70 by SIRT1 enhances Ku70-dependent DNA repair and inhibits mitochondrial apoptosis after genotoxic stimuli (Jeong et al., 2007; Sawada et al., 2003;). Also, SIRT1-mediated deacetylation of Krüppel associated box (KRAB)-associated protein 1 (KAP1) enhances the interaction between KAP1 and p53-binding protein 1 (53BP1) and NHEJ repair pathway (Lin et al., 2015). Finally, SIRT1 can regulate nucleotide excision repair (NER) by deacetylating and activating xeroderma pigmentosum $A$ and $C$ proteins (XPA and XPC) upon UV damage. Deacetylated XPA and XPC recognize DNA SSBs and recruit other NER factors at the breaks for DNA repair (Fan and Luo, 2010; Ming et al., 2010). Taken together, these studies support an essential role for SIRT1 as a tumor suppressor by improving genome stability.

SIRT6 functions as $\mathrm{NAD}^{+}$-dependent deacetylase and ADPribosyltransferase (Liszt et al., 2005; Michishita et al., 2008). Interestingly, SIRT6 knockout (KO) mice exhibit striking premature aging phenotypes such as lymphopenia, reduced subcutaneous fat, lordokyphosis and severe metabolic defects (Mostoslavsky et al., 2006). Because SIRT6 associates with telomere and deacetylates histone $\mathrm{H} 3 \mathrm{~K} 9$, which enhances the association of WRN protein at these regions and maintains proper telomeric chromatin (Michishita et al., 2008), defects of telomere functions in SIRT6 KO mice likely accounts for the premature aging phenotypes. SIRT6 KO mice also exhibited hypersensitivity to DNA damage and genomic instability, implying that SIRT6 has an important role in the DDR (Lombard et al. 2008; Mostoslavsky et al., 2006). Indeed, like SIRT1, SIRT6 is involved in several DNA repair pathways such as base excision repair (BER), HR and NHEJ. After DNA damage, SIRT6 has been reported to bind to DNA DSBs and enhance HR DNA repair through C-terminal binding protein (CtBP) interacting protein (CtIP) deacetylation (Kaidi et al., 2010). SIRT6 enhances NHEJ DNA repair by stabilizing DNA-PK, which senses DNA DSBs and promotes DNA repair (McCord et al., 2009). Recent studies also showed that upon oxidative DNA damage, SIRT6 associates with PARP1 and stimulates its activity through ADP-ribosylation, promoting NHEJ and HR DNA repair (Mao et al., 2011). Thus, these results suggest that SIRT6 might function as a tumor suppressor by inhibiting genetic instability.

SIRT7 is localized to the nucleolus as well as the nucleus and has histone deacetylase activity (Morris, 2013). SIRT7 regulates ribosomal DNA transcription in part by activating RNA polymerase 1 (Ford et al., 2006). Interestingly, recent studies suggested that SIRT7 has tumor promoting activities. Barber et al. (2012). showed that SIRT7 plays a critical role in maintaining oncogenic transformation by repressing transcription of genes involved in anchorage-independent- and contact inhibited-cell growth. In support of this notion, SIRT7 expression is highly increased in human hepatocellular carcinoma and SIRT7 loss results in the suppression of tumor cell growth (Kim et al., 2013). In addition, many other studies have suggested a critical role of SIRT7 in genotoxic stress resistance and cell survival. SIRT7 overexpression protects tumor cells against doxorubicininduced cell death (Kiran et al., 2015) and conversely, SIRT7 KO MEFs are resistant to oxidative DNA damage (Vakhrusheva et al., 2008a). SIRT7 is also known to directly deacetylate and hyperactivate the p53 tumor suppressor in vitro and in vivo, and Sirt7-deficient primary cardiomyocytes show a significantly diminished resistance to genotoxic stress (Vakhrusheva et al., 2008b). Interestingly, Mao et al. showed that, like SIRT6, SIRT7 overexpression enhances NHEJ and HR DNA repair after paraquat treatment (Mao et al., 2011), raising the possibility that SIRT7 may play a oncogenic role by protecting tumor cells against genotoxic stresses. 
The Roles of Sirtuins in Cancer

Seung Min Jeong \& Marcia C. Haigis

Table 1. Mouse models of sirtuins

\begin{tabular}{|c|c|c|c|}
\hline Sirtuin & Mouse models & Phenotypes & Role in tumorigenesis \\
\hline \multirow[t]{6}{*}{ SIRT1 } & $\mathrm{Tg}$ & Reduced incidence of spontaneous carcinomas and sarcomas & Suppression \\
\hline & $\mathrm{SIRT} 1^{\mathrm{Tg}} \mathrm{p} 53^{+/-}$ & Reduced overall tumor incidence in $\mathrm{p} 53^{+/-}$background & Suppression \\
\hline & $\mathrm{SIRT} 1^{\mathrm{Tg}} \mathrm{APC} \mathrm{min} /+^{-}$ & Inhibtion of intestinal tumors & Suppression \\
\hline & $\mathrm{SIRT} 1^{+/-} \mathrm{p} 53^{+/-}$ & Mammary tumors & Suppression \\
\hline & KO & Inhibition of chronic myelogenous leukemia development & Promotion \\
\hline & SIRT1 ${ }^{\text {Tg }}$ PTEN ${ }^{+/-}$ & Increased thyroid and prostate carcinomas & Promotion \\
\hline SIRT2 & KO & Mammary tumors (female), and liver and intestinal tumors (male) & Suppression \\
\hline SIRT3 & $\mathrm{KO}$ & Spontaneous mammary gland tumors & Suppression \\
\hline SIRT4 & KO & Spontaneous lung tumors and hyperinsulinemia & Suppression \\
\hline SIRT5 & $\mathrm{KO}$ & Defect in urea cycle and hyperammonemia & Unknown \\
\hline \multirow[t]{2}{*}{ SIRT6 } & KO & Premature aging phenotypes and short life span ( 3 weeks) & Unknown \\
\hline & SIRT6 ${ }^{\mathrm{flfl}} \mathrm{APC} \mathrm{min}^{\mathrm{mi}+}$ & Increased intestinal tumors & Suppression \\
\hline SIRT7 & KO & Premature aging phenotypes and short life span ( 1 year) & Unknown \\
\hline
\end{tabular}

SIRT2 is primarily localized in cytoplasm and functions as a cell cycle regulator (Dryden et al., 2003). SIRT2 regulates acetylation levels of $\mathrm{H} 4 \mathrm{~K} 16$ during mitosis, enabling chromatin compaction (Vaquero et al., 2006). SIRT2 overexpression induces a prolonged cell cycle, while downregulation inhibits cell cycle progression and induces cell cycle arrest (Dryden et al., 2003; North and Verdin, 2007). As an important cell cycle regulator, SIRT2 is required for genome maintenance by modulating the anaphase-promoting complex/cyclosome activity through deacetylation of CDH1 and CDC20 (Kim et al., 2011). SIRT2 loss causes accumulation of mitotic regulators such as the Aurora kinases that drive mitotic defects, resulting in centrosome amplification, aneuploidy and cell death. Also, SIRT2 KO mice (Table 1) develop mammary tumors and/or hepatocellular carcinomas (HCC) (Kim et al., 2011). Consistent with this idea, a recent study directly illustrates the importance of SIRT2 in cell cycle and genome stability. Serrano et al. showed that SIRT2 loss results in significant defects in cell cycle regulation and genome stability associated with defective monomethylation of H4K20 (Serrano et al., 2013). These studies imply that SIRT2 plays an important role in genome integrity by controlling cell cycle progression and DNA replication.

\section{In the mitochondria}

SIRT3, SIRT4 and SIRT5 are located in the mitochondria and have been implicated in mitochondria functions. SIRT3 is a major mitochondrial deacetylase that targets many enzymes involved in metabolism (Verdin et al., 2010). Beyond metabolic targets, SIRT3 has been shown to regulate the production of reactive oxygen species (ROS) from mitochondria by multiple mechanisms. For example, SIRT3 deacetylates and activates isocitrate dehydrogenase 2 (IDH2) and manganese superoxide dismutase (MnSOD) (Qiu et al., 2010; Tao et al., 2010), which maintain cellular ROS homeostasis. SIRT3 also deacetylates numerous components of the electron transport chain, suggesting that SIRT3 could directly suppress ROS production (Bell and Guarente, 2011). In this regard, SIRT3 loss increases cellular ROS levels, contributing to genomic and mitochondrial DNA instability, and SIRT3 KO mice develop estrogen receptor and progesterone receptor-positive mammary tumors (Kim et al., 2010a). Importantly, SIRT3 can bind and deacetylate Ku70 in response to DNA damage (Sundaresan et al., 2008), suggesting that SIRT3 might be involved in Ku70-dependent DNA repair.

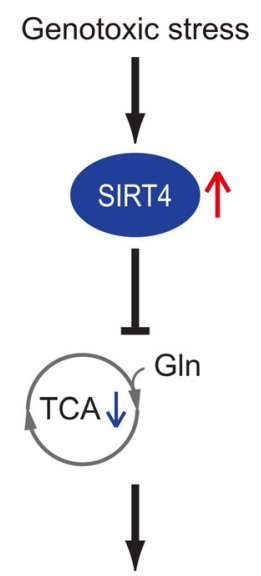

Cell cyle arrest DNA repair

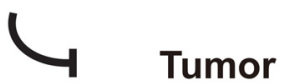

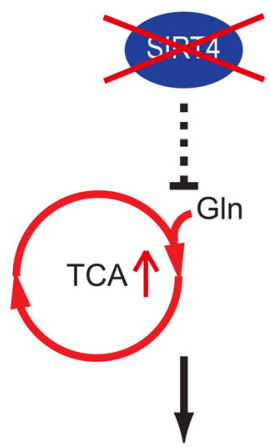

Tumor growth Genomic instability
Fig. 1. The regulation of metabolic response to DNA damage by SIRT4. DNA damage-induced SIRT4 represses the metabolism of glutamine (GIn) into TCA cycle, which contributes to the control of cell cycle progression and the maintenance of genomic integrity. Loss of SIRT4 leads to both increased glutaminolysis and DNA damage-induced genomic instability, resulting in tumorigenesis.

As discussed above, DNA damage elicits signaling pathways that initiate cell cycle arrest and DNA repair. However, the cellular metabolic response to DNA damage is not well elucidated. Recently, we found that SIRT4 functions as a unique "metabolic block" upon genotoxic stress (Jeong et al., 2013). SIRT4 is the most highly induced sirtuins in response to DNA damage stimuli and represses glutamine consumption without affecting glucose uptake, resulting in a decrease in the incorporation of glutamine into the tricarboxylic acid (TCA) cycle intermediates. This metabolic response contributes to cell cycle arrest of damaged cells and promotes the repair of damaged DNA. Indeed, loss of 
SIRT4 impaired DNA damage-induced cell cycle arrest and resulted in accumulation of DNA damage, and SIRT4 KO MEFs possessed more aneuploidy and exhibited an increased genomic instability (Fig. 1). Additionally, SIRT4 expression is decreased in many human tumors and, importantly, two independently derived SIRT4 KO mice spontaneously develop several types of tumors, most frequently lung tumors (Jeong et al., 2013). These findings identify SIRT4 as a tumor suppressor connecting two hallmarks of cancer: genomic instability and dysregulation of cell metabolism, suggesting that targeting of this link may be clinically beneficial.

SIRT5 is one of the least-studies mitochondrial sirtuins. It has been reported that SIRT5 enhances the urea cycle to detoxify ammonia by deacetylating carbamoyl phosphate synthetase 1 (CPS1), and Sirt5-deficent mice display high ammonia levels in blood under fasting or high protein diet (Nakagawa et al., 2009). However, except for its role in the urea cycle, SIRT5 KO mice did not exhibit any phenotypes and the physiological significance of SIRT5 in genomic maintenance is largely unknown.

\section{SIRTUINS IN CELL METABOLISM}

Reprogramming of cellular energy metabolism is a pivotal hallmark of cancer (Hanahan and Weinberg, 2011). Tumor cells often display elevated glycolysis and glutaminolysis to support their continued growth and survival. Enhanced glycolysis contributes to the bioenergetic needs of cancer cells and thus facilitates rapid cell proliferation (Tong et al., 2009). In addition, cancer cells also use critical intermediates derived from TCA cycle for the biosynthesis of nucleic acids, fatty acids and NAPDH to support their growth. Thus, replenishment of the mitochondrial carbon pool by increased glutamine metabolism is essential for the maintenance of mitochondrial integrity and its biosynthetic roles in cancer cells (DeBerardinis et al., 2007; Wise and Thompson, 2010). On the other hand, many cancer cells show alterations in lipid metabolism, which contributes to the synthesis of membranes and signaling molecules, and energy homeostasis in cancer cells (Currie et al., 2013; Santos and Schulze, 2012)

In addition to their roles in sustaining genomic stability, sirtuins are recognized as crucial regulators of cell metabolism. Sirtuins target many enzymes involved in central metabolism (Verdin et al., 2010) and the activities of sirtuins are controlled by cellular metabolic status through sensing changes of intracellular $\mathrm{NAD}^{+}$levels (Haigis and Guarente, 2006). Indeed, many studies have shown that sirtuins regulate glucose, glutamine and lipid metabolism (Guarente, 2013; Houtkooper et al., 2012; Martinez-Pastor and Mostoslavsky, 2012), implying that sirtuins may be implicated in tumor cell metabolism.

\section{In the nucleus and cytoplasm}

SIRT1 deacetylates a growing number of substrates that are involved in a wide variety of glucose metabolism. Peroxisome proliferator-activated receptor gamma coactivator $1 \alpha(P G C-1 \alpha)$, a master transcriptional co-activator of a number of genes, is a well-known target of SIRT1. In the liver, activated SIRT1 during fasting increases the deacetylation of PGC-1 $\alpha$, promoting the induction of gluconeogenic genes (Rodgers et al., 2005). In this regard, SIRT1 reinforces gluconeogenesis by activating a forkhead box protein $\mathrm{O} 1$ (FOXO1) transcription factor and peroxisome proliferators-activated receptor $\gamma$ (PPAR $\gamma$ ) through deacetylation (Frescas et al., 2005; Houtkooper et al., 2012). Additionally, SIRT1 inhibits glycolysis by repressing the expression of glycolytic genes through deacetylation of hypoxia-inducible factor $1 \alpha(\mathrm{HIF}-1 \alpha)$, and by directly inhibiting glycolytic enzyme phosphoglycerate mutase 1 (PGAM1) through deacetylation (Hallows et al., 2012; Lim et al., 2010). SIRT1 is also involved in glucose metabolism by positively regulating insulin secretion from pancreatic $\beta$ cells through uncoupling protein (UCP) 2 (Bordone et al., 2006; Moynihan et al., 2005).

On the other hand, SIRT1 plays an important role in lipid metabolism. During fasting, deacetylated PGC-1 $\alpha$ by SIRT1 activates PPAR $\alpha$, which promotes fatty-acid oxidation (FAO) by increasing the expression of related genes in muscle (GerhartHines et al., 2007). The sterol regulatory element-binding protein (SREBP) family has essential roles in lipid homeostasis. It has been shown that SIRT1 deacetylates SREBP-1c to stimulate fat oxidation under fasting condition (Ponugoti et al., 2010). Moreover, SIRT1 can interact with liver $X$ receptor (LXR) proteins, an upstream regulator of SREBP-1c, and activates it (Li et al., 2007). SIRT1 also induces fat metabolism and reduces fat storage in white adipose tissue by inhibiting PPAR $\gamma$ (Picard et al., 2004), facilitating fat catabolism. Thus, it appears that the expression and activity of SIRT1 are induced in several tissues during fasting or low nutrient conditions, which coordinates metabolic shift toward increased lipid metabolism for the organism's need for energy.

AMP-activated protein kinase (AMPK), a central energy sensor, has a key role in the maintaining cellular energy homeostasis. SIRT1 is also tightly related to AMPK activity, since SIRT1 activates the AMPK activator LKB1 through deacetylation (Lan et al., 2008) and AMPK increases NAMPT, a key NAD synthetic enzyme. (Canto et al., 2009). It has been shown that AMPK is involved in DDR. For example, in response to DNA damage, ATM phosphorylates and activates LKB1, activating AMPK (Fu et al., 2008; Sapkota et al., 2002). Activated AMPK stimulated mitochondrial biogenesis, and regulates DDR by interacting with p53 (Alexander and Walker, 2011). In line with these, Liu et al. showed that SIRT1 regulates DNA replication and DDR by sensing glucose deprivation and DNA damage through topoisomerase II binding protein1 (TopBP1) (Liu et al., 2014). These suggested that SIRT1 may play important roles in regulating energy metabolism in response to cellular stresses.

In addition to its essential roles in genomic stability, SIRT6 also functions as a critical regulator of glucose homeostasis. SIRT6 modulates glucose metabolism by maintaining deacetylation of H3K9 in promoters of glycolytic genes and functioning as a co-repressor of HIF-1 $\alpha$ (Zhong et al., 2010). Thus, SIRT6 loss shift cellular metabolism toward increased glycolysis, a metabolic reprogramming similar to the Warburg effect. In mice, SIRT6 loss enhances the expression of GLUT1, the glucose transporter, leading to increased glucose uptake, and SIRT6 KO mice die early caused by a severe hypoglycemia (Mostoslavsky et al., 2006). In this regard, one recent study proved that SIRT6 functions as a tumor suppressor by repressing aerobic glycolysis, and showed that conditional SIRT6 KO mice exhibited increased tumorigenesis (Sebastian et al., 2012). Moreover, SIRT6 expression was decreased in human cancers such as pancreatic cancer and colon carcinoma (Sebastian et al., 2012), indicating that SIRT6 functions as a tumor suppressor acting to suppress aerobic glycolysis. With regard to glucose homeostasis, SIRT6 is involved in gluconeogenesis. SIRT6-mediated deacetylation of general control nonrepressed protein 5 (GCN5) induces the acetylation of PGC-1 $\alpha$ acetylation and suppresses hepatic glucose production (Dominy et al., 2012).

Other data suggest that SIRT6 also regulates fat utilization. Mice with a liver-specific deletion of SIRT6 exhibit a fatty liver 
and hepatocytes from these mice show decreased FAO (Kim et al., 2010b), while SIRT6 overexpression protects mice against high-fat diet (HFD) induced fat accumulation (Kanfi et al., 2010).

Compared to its premature aging phenotypes, including a reduction of life span, heart hypertrophy, loss of subcutaneous fat and degenerative cardiac hypertrophy (Vakhrusheva et al., 2008b), the metabolic functions of SIRT7 are emerging. Interestingly, it was reported that SIRT7 functions as a negative regulator of HIF signaling. SIRT7 knockdown results in increased transcriptional activities of HIF-1 and HIF-2 in several cancer cell lines (Hubbi et al., 2013), implying that SIRT7 might be involved in cancer metabolism. Recent studies point to a link between SIRT7 and lipid metabolism. SIRT7 KO mice exhibit chronic hepatosteatosis (Shin et al., 2013) and liver-specific deletion of SIRT7 also develop hepatic microvesicular steatosis (Ryu et al., 2014). Opposite to these results, another report showed that SIRT7-deficient mice are resistant to HFD-induced hepatic steatosis and obesity (Yoshizawa et al., 2014). Thus, future studies should be undertaken to uncover the functions and molecular mechanisms of SIRT7 in metabolism and tumorigenesis.

In response to cellular stress and calorie restriction, SIRT2 deacetylates FOXO1 and FOXO3a (Jing et al., 2007; Wang et al., 2007) that modulates glucose and lipid metabolism, suggesting that SIRT2 is implicated in metabolic homeostasis. Indeed, it has been showed that SIRT2 promotes gluconeogenesis by deacetylating the rate-limiting enzyme phosphoenolpyruvate carboxykinase (PEPCK), thereby modulating cellular response to glucose (Jiang et al., 2011). In addition, transcriptional repression of SIRT2 by HIF- $1 \alpha$ leads to compromised PGC1 $\alpha$ deacetylation, which results in reduction of $\beta$-oxidation and thus the development of obesity (Krishnan et al., 2012). At the same time, SIRT2 destabilizes HIF-1 $\alpha$ through deacetylation and regulates tumor metabolism under hypoxic conditions (Seo et al., 2015).

\section{In the mitochondria}

Because of their localization, mitochondrial sirtuins have been expected to play key roles as sensors and regulators of energy status in this organelle. SIRT3 has been shown to bind and regulate multiple enzymes for metabolic homeostasis. For example, during low nutrient conditions, SIRT3 plays an essential role for maintenance of energy homeostasis by deacetylating mitochondrial proteins (Hebert et al., 2013). SIRT3 targets several components of the TCA cycle and electron transport chain including NADH dehydrogenase (ubiquinone) 1 Alpha subcomplex 9 (NDUF9) (Ahn et al., 2008), IDH2 (Someya et al., 2010) and ATP synthase (Anderson and Hirschey, 2012). Moreover, SIRT3 can promote mitochondrial FAO by deacetylating long-chain acyl coenzyme A dehydrogenase (LCAD) (Hallows et al., 2011; Hirschey et al., 2010) to stimulate alternative energy production pathways during fasting. In addition, SIRT3 also enhances ketone body production by activating 3hydroxy-3-methylglutaryl-CoA synthase (HMGCS2) through deacetylation (Shimazu et al., 2010).

Recently, we and others demonstrated that SIRT3 loss shifts cellular metabolism toward enhanced glycolysis, a common metabolic switch in many cancer cells (Vander Heiden et al., 2009). SIRT3 mediates this metabolic reprogramming through HIF-1 $\alpha$, a key transcription factor that induces a lot of glycolytic genes (Bell et al., 2011; Finley et al., 2011). SIRT3 loss increases ROS, leading to HIF-1 $\alpha$ stabilization. As a consequence, SIRT3 KO MEFs exhibit both enhanced glycolysis and increased glucose-dependent cell proliferation (Finley et al.,
2011). SIRT3 is also involved in tumor suppression by regulating iron homeostasis. SIRT3 loss contributes to tumor growth by increasing cellular iron metabolism, which is essential for DNA synthesis, oxygen transport and respiration (Jeong et al., 2015).

Previously, it has been shown that SIRT4 regulates glutamine metabolism in the pancreas by inhibiting mitochondrial glutamate dehydrogenase (GDH) which converts glutamate to the TCA cycle intermediates $\alpha$-ketoglutarate and ammonia (Ahuja et al., 2007; Haigis et al., 2006). Recently, we and others reported that SIRT4 exerts tumor suppressive activities by repressing mitochondrial glutamine metabolism, in part through modification and repression of GDH (Csibi et al., 2013; Jeong et al., 2013). SIRT4 loss enhances glutamine anaplerosis and glutamine-dependent cell proliferation, while SIRT4 overexpression inhibits glutamine metabolism and sensitizes tumor cells to glucose depletion. In this regard, the tumor suppressive role of SIRT4 was further evaluated in the context of Mycinduced B cell lymphomagenesis (Jeong et al., 2014). SIRT4 overexpression inhibits glutamine utilization and proliferation of human Burkitt lymphoma cells. Moreover, SIRT4 loss in a mouse Burkitt lymphoma model, E $\mu-M y c$ transgenic mouse, accelerates lymphomagenesis and mortality. Importantly, SIRT4 is involved in cellular ammonia production. Ammonia is produced as a byproduct of glutamine catabolism in the mitochondria and it has been reported that in glioblastoma cells, most of the ammonia (>90\%) in the media was derived from this glutamine anaplerosis (Yang et al., 2009). Thus, SIRT4-mediated reduction of glutamine consumption was accompanied by a reduction in ammonia production, and SIRT4 KO cells produce more ammonia (Jeong et al., 2013; 2014). Because ammonia derived from glutaminolysis has been known as a regulator of autophagy (Eng et al., 2010), it will be interesting whether SIRT4 affects autophagic flux in cancer cells.

SIRT4 was known to have NAD ${ }^{+}$-dependent mono-ADP ribosyltranferase activity without recognized deacetylase activity (Haigis et al., 2006). Interestingly, our recent study proved that SIRT4 can coordinate cellular lipid metabolism by repressing malonyl CoA decarboxylase (MCD) through deacetylation (Laurent et al., 2013). In mice, SIRT4 represses FAO and promotes lipid anabolism under nutrient-replete conditions, and thus SIRT4 KO mice exhibit deregulated lipid metabolism, increased exercise tolerance and protection against diet-induced obesity (Laurent et al., 2013). The fact that SIRT4 appears to have important roles in FAO suggests that SIRT4 may regulate tumor cell growth by limiting fat utilization.

As previously mentioned, an early study showed that SIRT5 regulates ammonia metabolism through the deacetylation of CPS1. Importantly, it has been reported that SIRT5 also possesses $\mathrm{NAD}^{+}$-dependent demalonylase and desuccinylase activities (Du et al., 2011). Further evidence is the finding that SIRT5 binds to glutaminase, the first enzyme for mitochondrial glutamine metabolism, and inhibits it through desuccinylation (Polletta et al., 2015). Given the existence of plenty of succinylation sites on proteins, involved in the TCA cycle, amino acid degradation and lipid metabolism (Park et al., 2013), SIRT5 is likely to possess other metabolic roles. Thus, future studies will need to dissect the role of SIRT5 in cancer metabolism in influencing acetylation and/or succinylation of target proteins.

\section{DOUBLE-EDGED ROLES OF SIRTUINS IN CANCER}

Sirtuins have merged as key regulators of genomic stability and metabolic homeostasis, contributing to stress resistance 
$\underline{\text { Genomic stability }}$

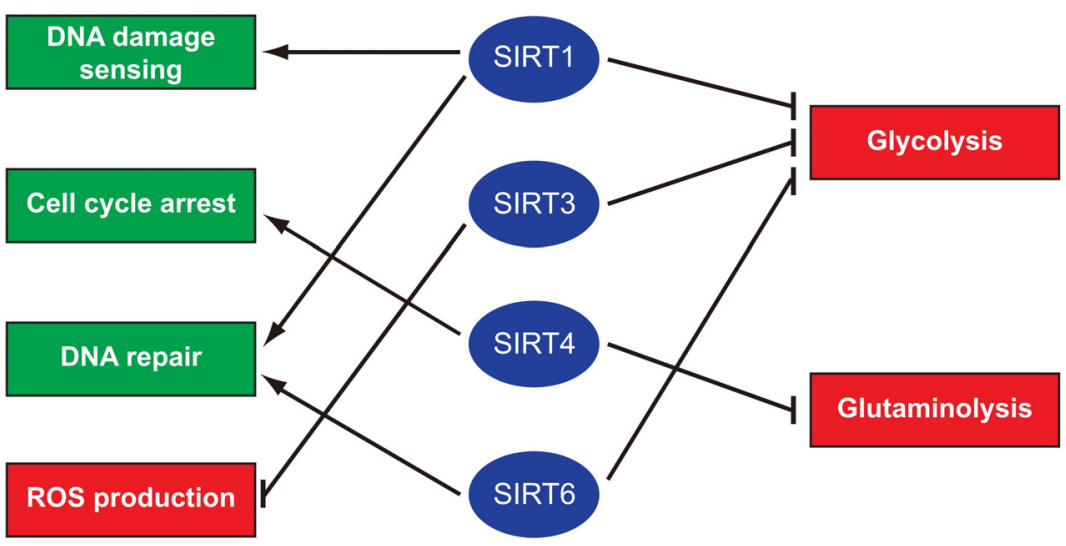

Fig. 2. Sirtuins regulate genomic stability and metabolism. The roles of sirtuins in the DDR and cell metabolism are summarized. Oncogenic and tumor suppressive pathways are displayed in red and green, respectively. and tumor suppression. However, as we discussed about the oncogenic functions of SIRT7, there is plenty of evidence suggesting that several sirtuins may promote tumor growth and survival. Several studies have demonstrated that the expression of SIRT1 is upregulated in various human cancers (Bradbury et al., 2005; Huffman et al., 2007; Lim, 2006; Wang et al., 2008). In line with these, SIRT1 overexpression promotes the incidence of thyroid and prostate carcinomas in PTENdeficiency mice (Herranz et al., 2013). Also, in another study, SIRT1 deletion suppresses BCR-ABL transformation of bone marrow cells and development of a CML-like myeloid disease (Yuan et al., 2012). Increased SIRT2 expression was also observed in certain types of cancer including acute myeloid leukemia (Dan et al., 2012), pancreatic cancer and neuroblastoma (Liu et al., 2013), and its expression is positively correlated with high-grade human HCC and prostate cancers (Chen et al., 2013; Hou et al., 2012). In addition, it was reported that SIRT3 expression is increased in several oral squamous cell carcinoma (OSCC) samples and downregulation of SIRT3 inhibits survival and proliferation of OSCC cells (Alhazzazi et al., 2011). Consistent with these findings, SIRT3 can promote the proliferation of bladder carcinoma cells by abrogating p53-induced growth arrest and senescence (Li et al., 2010), and protect cancer cells against DNA damage-induced cell death through Ku70 deacetylation (Sundaresan et al., 2008). Finally, SIRT6 can function as an oncogene by promoting cancer-induced inflammation, angiogenesis and metastasis in pancreatic cancer cells (Bauer et al., 2012), and contributing cancer cell survival against chemotherapeutic agents through the inhibition of FOXO3a activity (Khongkow et al., 2013).

It has been known that many genes, which have tumor suppressive activities, can function as oncogenes depend on genetic context, tumor type and stage. Because sirtuins function as key regulators of cellular homeostasis, they may serve as barriers to inhibit tumor initiation. However, on the other hand, tumor cells take advantage of sirtuins to contribute to their growth and survival by enhancing stress resistance and modulating cell metabolism. Thus, further studies are needed for delineating the roles of sirtuins in cancer.

\section{CONCLUSION}

There has been a tremendous amount of advances in our un- derstanding of the role of sirtuins in cancer. Growing evidence supports that sirtuins are clearly involved in regulating genome integrity and metabolic processes (Fig. 2), and link these two hallmarks of cancer. However, the roles of sirtuins in tumorigenesis are complex and may function as both tumor suppressor and oncogene. Thus, further work will be needed to pinpoint the exact molecular mechanisms that enabling the role of each sirtuin in cancer. Also, it remains to be determined whether sirtuins plays an important role in tumor invasion and metastasis. Last, the delineation of the interaction between sirtuins during tumorigenesis will allow for a deeper understanding of how they coordinately regulate genomic fidelity and metabolism of cancer.

\section{ACKNOWLEDGMENTS}

We apologize for the many studies that were not discussed because of limited space. S.M.J. was supported by the Catholic Medical Center Research Foundation made in the program year of 2015. M.C.H. was supported by an American Cancer Society Research Scholar Grant, the National Institutes of Health (NIH) Grant AG032375, and the Glenn Foundation for Medical Research.

\section{REFERENCES}

Abbas, T., and Dutta, A. (2009). p21 in cancer: intricate networks and multiple activities. Nat. Rev. Cancer 9, 400-414.

Abraham, R.T. (2001). Cell cycle checkpoint signaling through the ATM and ATR kinases. Genes Dev. 15, 2177-2196.

Ahn, B.H., Kim, H.S., Song, S., Lee, I.H., Liu, J., Vassilopoulos, A., Deng, C.X. and Finkel, T. (2008). A role for the mitochondrial deacetylase Sirt3 in regulating energy homeostasis. Proc. Natl. Acad. Sci. USA 105, 14447-14452.

Ahuja, N., Schwer, B., Carobbio, S., Waltregny, D., North, B.J., Castronovo, V., Maechler, P. and Verdin, E. (2007). Regulation of insulin secretion by SIRT4, a mitochondrial ADPribosyltransferase. J. Biol. Chem. 282, 33583-33592.

Alexander, A., and Walker, C.L. (2011). The role of LKB1 and AMPK in cellular responses to stress and damage. FEBS Lett. 585, 952-957.

Alhazzazi, T.Y., Kamarajan, P., Joo, N., Huang, J.Y., Verdin, E., D'Silva, N.J., and Kapila, Y.L. (2011). Sirtuin-3 (SIRT3)., a novel potential therapeutic target for oral cancer. Cancer 117, 16701678.

Anderson, K.A., and Hirschey, M.D. (2012). Mitochondrial protein acetylation regulates metabolism. Essays Biochem. 52, 23-35. 
Barber, M.F., Michishita-Kioi, E., Xi, Y., Tasselli, L., Kioi, M., Moqtaderi, Z., Tennen, R.I., Paredes, S., Young, N.L., Chen, K., et al. (2012). SIRT7 links H3K18 deacetylation to maintenance of oncogenic transformation. Nature 487, 114-118.

Bauer, I., Grozio, A., Lasiglie, D., Basile, G., Sturla, L., Magnone, M., Sociali, G., Soncini, D., Caffa, I., Poggi, A., et al. (2012). The NAD+-dependent histone deacetylase SIRT6 promotes cytokine production and migration in pancreatic cancer cells by regulating Ca2+ responses. J. Biol. Chem. 287, 40924-40937.

Bell, E.L., and Guarente, L. (2011). The SirT3 divining rod points to oxidative stress. Mol. Cell 42, 561-568.

Bell, E.L., Emerling, B.M., Ricoult, S.J., and Guarente, L. (2011). SirT3 suppresses hypoxia inducible factor 1alpha and tumor growth by inhibiting mitochondrial ROS production. Oncogene 30, 2986-2996.

Bordone, L., Motta, M.C., Picard, F., Robinson, A., Jhala, U.S., Apfeld, J., McDonagh, T., Lemieux, M., McBurney, M., Szilvasi, A., et al. (2006). Sirt1 regulates insulin secretion by repressing UCP2 in pancreatic beta cells. PLoS Biol. 4, e31

Bradbury, C.A., Khanim, F.L., Hayden, R., Bunce, C.M., White, D.A., Drayson, M.T., Craddock, C., and Turner, B.M. (2005). Histone deacetylases in acute myeloid leukaemia show a distinctive pattern of expression that changes selectively in response to deacetylase inhibitors. Leukemia 19, 1751-1759.

Canto, C., Gerhart-Hines, Z., Feige, J.N., Lagouge, M., Noriega, L., Milne, J.C., Elliott, P.J., Puigserver, P., and Auwerx, J. (2009). AMPK regulates energy expenditure by modulating $\mathrm{NAD}^{+}$ metabolism and SIRT1 activity. Nature 458, 1056-1060.

Chen, J., Chan, A.W., To, K.F., Chen, W., Zhang, Z., Ren, J., Song, C., Cheung, Y.S., Lai, P.B., Cheng, S.H., et al. (2013). SIRT2 overexpression in hepatocellular carcinoma mediates epithelial to mesenchymal transition by protein kinase B/glycogen synthase kinase-3beta/beta-catenin signaling. Hepatology 57, 2287-2298.

Ciccia, A., and Elledge, S.J. (2010). The DNA damage response: making it safe to play with knives. Mol. Cell 40,179-204.

Cosentino, C., Grieco, D., and Costanzo, V. (2011). ATM activates the pentose phosphate pathway promoting anti-oxidant defence and DNA repair. EMBO J. 30, 546-555.

Csibi, A., Fendt, S.M., Li, C., Poulogiannis, G., Choo, A.Y., Chapski, D.J., Jeong, S.M., Dempsey, J.M., Parkhitko, A., Morrison, T., et al. (2013). The mTORC1 pathway stimulates glutamine metabolism and cell proliferation by repressing SIRT4. Cell 153, 840-854.

Currie, E., Schulze, A., Zechner, R., Walther, T.C., and Farese, R.V., Jr. (2013). Cellular fatty acid metabolism and cancer. Cell Metabol. 18, 153-161.

Dan, L., Klimenkova, O., Klimiankou, M., Klusman, J.H., van den Heuvel-Eibrink, M.M., Reinhardt, D., Welte, K., and Skokowa, J. (2012). The role of sirtuin 2 activation by nicotinamide phosphoribosyltransferase in the aberrant proliferation and survival of myeloid leukemia cells. Haematologica 97, 551-559.

Dang, C.V. (2010). Rethinking the Warburg effect with Myc micromanaging glutamine metabolism. Cancer Res. 70, 859862.

DeBerardinis, R.J., Mancuso, A., Daikhin, E., Nissim, I., Yudkoff, M., Wehrli, S., and Thompson, C.B. (2007). Beyond aerobic glycolysis: transformed cells can engage in glutamine metabolism that exceeds the requirement for protein and nucleotide synthesis. Proc. Natl. Acad. Sci. USA 104, 1934519350.

Deng, C.X. (2006). BRCA1: cell cycle checkpoint, genetic instability, DNA damage response and cancer evolution. Nucleic Acids Res. 34, 1416-1426.

Dominy, J.E., Jr., Lee, Y., Jedrychowski, M.P., Chim, H., Jurczak, M.J., Camporez, J.P., Ruan, H.B., Feldman, J., Pierce, K., Mostoslavsky, R., et al. (2012). The deacetylase Sirt6 activates the acetyltransferase GCN5 and suppresses hepatic gluconeogenesis. Mol. Cell 48, 900-913.

Dryden, S.C., Nahhas, F.A., Nowak, J.E., Goustin, A.S., and Tainsky, M.A. (2003). Role for human SIRT2 NAD-dependent deacetylase activity in control of mitotic exit in the cell cycle. Mol. Cell. Biol. 23, 3173-3185.

Du, J., Zhou, Y., Su, X., Yu, J.J., Khan, S., Jiang, H., Kim, J., Woo, J., Kim, J.H., Choi, B.H., et al. (2011). Sirt5 is a NAD-dependent protein lysine demalonylase and desuccinylase. Science 334 ,
806-809.

Eng, C.H., Yu, K., Lucas, J., White, E., and Abraham, R.T. (2010). Ammonia derived from glutaminolysis is a diffusible regulator of autophagy. Sci. Signal. 3, ra31.

Fan, W., and Luo, J. (2010). SIRT1 regulates UV-induced DNA repair through deacetylating XPA. Mol. Cell 39, 247-258.

Finkel, T., Deng, C.X., and Mostoslavsky, R. (2009). Recent progress in the biology and physiology of sirtuins. Nature 460 , 587-591.

Finley, L.W., Carracedo, A., Lee, J., Souza, A., Egia, A., Zhang, J., Teruya-Feldstein, J., Moreira, P.I., Cardoso, S.M., Clish, C.B., et al. (2011). SIRT3 opposes reprogramming of cancer cell metabolism through HIF1alpha destabilization. Cancer Cell 19, 416-428

Ford, E., Voit, R., Liszt, G., Magin, C., Grummt, I., and Guarente, L. (2006). Mammalian Sir2 homolog SIRT7 is an activator of RNA polymerase I transcription. Genes Dev. 20, 1075-1080.

Frescas, D., Valenti, L., and Accili, D. (2005). Nuclear trapping of the forkhead transcription factor FoxO1 via Sirt-dependent deacetylation promotes expression of glucogenetic genes. J. Biol. Chem. 280, 20589-20595

Fu, X., Wan, S., Lyu, Y.L., Liu, L.F., and Qi, H. (2008). Etoposide induces ATM-dependent mitochondrial biogenesis through AMPK activation. PLoS One 3, e2009.

Gatenby, R.A., and Gillies, R.J. (2004). Why do cancers have high aerobic glycolysis? Nat. Rev. Cancer 4, 891-899.

Gerhart-Hines, Z., Rodgers, J.T., Bare, O., Lerin, C., Kim, S.H., Mostoslavsky, R., Alt, F.W., Wu, Z., and Puigserver, P. (2007) Metabolic control of muscle mitochondrial function and fatty acid oxidation through SIRT1/PGC-1alpha. EMBO J. 26, 1913-1923.

Guarente, L. (2013). Calorie restriction and sirtuins revisited. Genes Dev. 27, 2072-2085.

Guarente, L., and Kenyon, C. (2000). Genetic pathways that regulate ageing in model organisms. Nature 408, 255-262.

Haigis, M.C., and Guarente, L.P. (2006). Mammalian sirtuins-emerging roles in physiology, aging, and calorie restriction. Genes Dev. 20, 2913-2921.

Haigis, M.C., and Sinclair, D.A. (2010). Mammalian sirtuins: biological insights and disease relevance. Ann. Rev. Pathol. 5 , 253-295.

Haigis, M.C., Mostoslavsky, R., Haigis, K.M., Fahie, K., Christodoulou, D.C., Murphy, A.J., Valenzuela, D.M., Yancopoulos, G.D., Karow, M., Blander, G., et al. (2006). SIRT4 inhibits glutamate dehydrogenase and opposes the effects of calorie restriction in pancreatic beta cells. Cell 126, 941-954.

Hallows, W.C., Yu, W., Smith, B.C., Devries, M.K., Ellinger, J.J., Someya, S., Shortreed, M.R., Prolla, T., Markley, J.L., Smith, L.M., et al. (2011). Sirt3 promotes the urea cycle and fatty acid oxidation during dietary restriction. Mol. Cell 41, 139-149.

Hallows, W.C., Yu, W., and Denu, J.M. (2012). Regulation of glycolytic enzyme phosphoglycerate mutase-1 by Sirt1 proteinmediated deacetylation. J. Biol. Chem. 287, 3850-3858.

Hanahan, D., and Weinberg, R.A. (2011). Hallmarks of cancer: the next generation. Cell 144, 646-674.

Hebert, A.S., Dittenhafer-Reed, K.E., Yu, W., Bailey, D.J., Selen, E.S., Boersma, M.D., Carson, J.J., Tonelli, M., Balloon, A.J., Higbee, A.J., et al. (2013). Calorie restriction and SIRT3 trigger global reprogramming of the mitochondrial protein acetylome. Mol. Cell 49, 186-199.

Herranz, D., Maraver, A., Canamero, M., Gomez-Lopez, G., Inglada-Perez, L., Robledo, M., Castelblanco, E., Matias-Guiu, X., and Serrano, M. (2013). SIRT1 promotes thyroid carcinogenesis driven by PTEN deficiency. Oncogene 32, 40524056.

Hirschey, M.D., Shimazu, T., Goetzman, E., Jing, E., Schwer, B., Lombard, D.B., Grueter, C.A., Harris, C., Biddinger, S., llkayeva, O.R., et al. (2010). SIRT3 regulates mitochondrial fatty-acid oxidation by reversible enzyme deacetylation. Nature 464,121 125.

Hou, H., Chen, W., Zhao, L., Zuo, Q., Zhang, G., Zhang, X., Wang, H., Gong, H., Li, X., Wang, M., et al. (2012). Cortactin is associated with tumour progression and poor prognosis in prostate cancer and SIRT2 other than HADC6 may work as facilitator in situ. J. Clin. Pathol. 65, 1088-1096.

Houtkooper, R.H., Pirinen, E., and Auwerx, J. (2012). Sirtuins as regulators of metabolism and healthspan. Nat. Rev. Mol. Cell 
Biol. 13, 225-238.

Hubbi, M.E, Hu, H. Kshitiz, Gilkes, D.M, and Semenza, G.L. (2013). Sirtuin-7 inhibits the activity of hypoxia-inducible factors. J. Biol. Chem. 288, 20768-20775

Huffman, D.M., Grizzle, W.E., Bamman, M.M., Kim, J.S., Eltoum, I.A., Elgavish, A., and Nagy, T.R. (2007). SIRT1 is significantly elevated in mouse and human prostate cancer. Cancer Res. 67, 6612-6618.

Jeong, J., Juhn, K., Lee, H., Kim, S.H., Min, B.H., Lee, K.M., Cho, M.H., Park, G.H., and Lee, K.H. (2007). SIRT1 promotes DNA repair activity and deacetylation of Ku70. Exp. Mol. Med. 39, 813.

Jeong, S.M., Xiao, C., Finley, L.W., Lahusen, T., Souza, A.L., Pierce, K., Li, Y.H., Wang, X., Laurent, G., German, N.J., et al. (2013). SIRT4 has tumor-suppressive activity and regulates the cellular metabolic response to DNA damage by inhibiting mitochondrial glutamine metabolism. Cancer Cell 23, 450-463.

Jeong, S.M., Lee, A., Lee, J., and Haigis, M.C. (2014). SIRT4 protein suppresses tumor formation in genetic models of Mycinduced B cell lymphoma. J. Biol. Chem. 289, 4135-4144.

Jeong, S.M., Lee, J., Finley, L.W., Schmidt, P.J., Fleming, M.D., and Haigis, M.C. (2015). SIRT3 regulates cellular iron metabolism and cancer growth by repressing iron regulatory protein 1 . Oncogene 34, 2115-2124.

Jiang, W., Wang, S., Xiao, M., Lin, Y., Zhou, L., Lei, Q., Xiong, Y., Guan, K.L., and Zhao, S. (2011). Acetylation regulates gluconeogenesis by promoting PEPCK1 degradation via recruiting the UBR5 ubiquitin ligase. Mol. Cell 43, 33-44.

Jing, E., Gesta, S., and Kahn, C.R. (2007). SIRT2 regulates adipocyte differentiation through FoxO1 acetylation/deacetylation. Cell Metabol. 6, 105-114.

Kaidi, A., Weinert, B.T., Choudhary, C., and Jackson, S.P. (2010). Human SIRT6 promotes DNA end resection through CtIP deacetylation. Science 329, 1348-1353.

Kanfi, Y., Peshti, V., Gil, R., Naiman, S., Nahum, L., Levin, E., Kronfeld-Schor, N., and Cohen, H.Y. (2010). SIRT6 protects against pathological damage caused by diet-induced obesity. Aging Cell 9, 162-173.

Khongkow, M., Olmos, Y., Gong, C., Gomes, A.R., Monteiro, L.J., Yague, E., Cavaco, T.B., Khongkow, P., Man, E.P., Laohasinnarong, S., et al. (2013). SIRT6 modulates paclitaxel and epirubicin resistance and survival in breast cancer Carcinogenesis 34, 1476-1486.

Kim, H.S., Patel, K., Muldoon-Jacobs, K., Bisht, K.S., Aykin-Burns, N., Pennington, J.D., van der Meer, R., Nguyen, P., Savage, J., Owens, K.M., et al. (2010a). SIRT3 is a mitochondria-localized tumor suppressor required for maintenance of mitochondrial integrity and metabolism during stress. Cancer Cell 17, 41-52.

Kim, H.S., Xiao, C., Wang, R.H., Lahusen, T., Xu, X., Vassilopoulos, A., Vazquez-Ortiz, G., Jeong, W.I., Park, O., Ki, S.H., et al. (2010b). Hepatic-specific disruption of SIRT6 in mice results in fatty liver formation due to enhanced glycolysis and triglyceride synthesis. Cell Metabol. 12, 224-236.

Kim, H.S., Vassilopoulos, A., Wang, R.H., Lahusen, T., Xiao, Z., Xu, X., Li, C., Veenstra, T.D., Li, B., Yu, H., et al. (2011). SIRT2 maintains genome integrity and suppresses tumorigenesis through regulating APC/C activity. Cancer Cell 20, 487-499.

Kim, J.K., Noh, J.H., Jung, K.H., Eun, J.W., Bae, H.J., Kim, M.G., Chang, Y.G., Shen, Q., Park, W.S., Lee, J.Y., et al. (2013). Sirtuin7 oncogenic potential in human hepatocellular carcinoma and its regulation by the tumor suppressors MiR-125a-5p and MiR-125b. Hepatology 57, 1055-1067.

Kiran, S., Oddi, V., and Ramakrishna, G. (2015). Sirtuin 7 promotes cellular survival following genomic stress by attenuation of DNA damage, SAPK activation and p53 response. Exp. Cell Res. 331, 123-141.

Krishnan, J., Danzer, C., Simka, T., Ukropec, J., Walter, K.M., Kumpf, S., Mirtschink, P., Ukropcova, B., Gasperikova, D., Pedrazzini, T., et al. (2012). Dietary obesity-associated Hif1alpha activation in adipocytes restricts fatty acid oxidation and energy expenditure via suppression of the Sirt2-NAD+ system. Genes Dev. 26, 259-270.

Lan, F., Cacicedo, J.M., Ruderman, N., and Ido, Y. (2008). SIRT1 modulation of the acetylation status, cytosolic localization, and activity of LKB1. Possible role in AMP-activated protein kinase activation. J. Biol. Chem. 283, 27628-27635.
Lapenna, S., and Giordano, A. (2009). Cell cycle kinases as therapeutic targets for cancer. Nat. Rev. Drug Discov. 8, 547-566. Laurent, G., German, N.J., Saha, A.K., de Boer, V.C., Davies, M., Koves, T.R., Dephoure, N., Fischer, F., Boanca, G., Vaitheesvaran, B., et al. (2013). SIRT4 coordinates the balance between lipid synthesis and catabolism by repressing malonyl CoA decarboxylase. Mol. Cell 50,686-698.

Li, X., Zhang, S., Blander, G., Tse, J.G., Krieger, M. and Guarente, L. (2007). SIRT1 deacetylates and positively regulates the nuclear receptor LXR. Mol. Cell 28, 91-106.

Li, K., Casta, A., Wang, R., Lozada, E., Fan, W., Kane, S., Ge, Q., Gu, W., Orren, D., and Luo, J. (2008). Regulation of WRN protein cellular localization and enzymatic activities by SIRT1mediated deacetylation. J. Biol. Chem. 283, 7590-7598.

Li, S., Banck, M., Mujtaba, S., Zhou, M.M., Sugrue, M.M., and Walsh, M.J. (2010). p53-induced growth arrest is regulated by the mitochondrial SirT3 deacetylase. PLoS One 5, e10486.

Lim, C.S. (2006). SIRT1: tumor promoter or tumor suppressor? Med. Hypotheses 67, 341-344.

Lim, J.H., Lee, Y.M., Chun, Y.S., Chen, J., Kim, J.E., and Park, J.W. (2010). Sirtuin 1 modulates cellular responses to hypoxia by deacetylating hypoxia-inducible factor 1alpha. Mol. Cell 38, 864878

Lin, Y.H., Yuan, J., Pei, H., Liu, T., Ann, D.K., and Lou, Z. (2015) KAP1 deacetylation by SIRT1 promotes non-homologous endjoining repair. PLoS One 10, e0123935.

Liszt, G., Ford, E., Kurtev, M., and Guarente, L. (2005). Mouse Sir2 homolog SIRT6 is a nuclear ADP-ribosyltransferase. J. Biol. Chem. 280, 21313-21320.

Liu, P.Y., Xu, N., Malyukova, A., Scarlett, C.J., Sun, Y.T., Zhang, X.D., Ling, D., Su, S.P., Nelson, C., Chang, D.K., et al. (2013) The histone deacetylase SIRT2 stabilizes Myc oncoproteins. Cell Death Differ. 20, 503-514

Liu, T., Lin, Y.H., Leng, W., Jung, S.Y., Zhang, H., Deng, M., Evans, D., Li, Y., Luo, K., Qin, B., et al. (2014). A divergent role of the SIRT1-TopBP1 axis in regulating metabolic checkpoint and DNA damage checkpoint. Mol. Cell 56, 681-695.

Lombard, D.B., Schwer, B., Alt, F.W., and Mostoslavsky, R. (2008) SIRT6 in DNA repair, metabolism and ageing. J. Int. Med. 263 128-141.

Mao, Z., Hine, C., Tian, X., Van Meter, M., Au, M., Vaidya, A., Seluanov, A., and Gorbunova, V. (2011). SIRT6 promotes DNA repair under stress by activating PARP1. Science 332, 14431446.

Martinez-Pastor, B., and Mostoslavsky, R. (2012). Sirtuins, metabolism, and cancer. Front. Pharmacol. 3, 22.

Mathias, R.A., Greco, T.M., Oberstein, A., Budayeva, H.G., Chakrabarti, R., Rowland, E.A., Kang, Y., Shenk, T., and Cristea, I.M. (2014). Sirtuin 4 is a lipoamidase regulating pyruvate dehydrogenase complex activity. Cell 159, 1615-1625.

McCord, R.A., Michishita, E., Hong, T., Berber, E., Boxer, L.D. Kusumoto, R., Guan, S., Shi, X., Gozani, O., Burlingame, A.L. et al. (2009). SIRT6 stabilizes DNA-dependent protein kinase at chromatin for DNA double-strand break repair. Aging 1, 109-121.

Michishita, E., McCord, R.A., Berber, E., Kioi, M., Padilla-Nash, H., Damian, M., Cheung, P., Kusumoto, R., Kawahara, T.L., Barrett, J.C., et al. (2008). SIRT6 is a histone H3 lysine 9 deacetylase that modulates telomeric chromatin. Nature 452, 492-496.

Ming, M., Shea, C.R., Guo, X., Li, X., Soltani, K., Han, W., and He, Y.Y. (2010). Regulation of global genome nucleotide excision repair by SIRT1 through xeroderma pigmentosum C. Proc. Natl. Acad. Sci. USA 107, 22623-22628.

Morris, B.J. (2013). Seven sirtuins for seven deadly diseases of aging. Free Radical Biol. Med. 56, 133-171.

Mostoslavsky, R., Chua, K.F., Lombard, D.B., Pang, W.W., Fischer, M.R., Gellon, L., Liu, P., Mostoslavsky, G., Franco, S., Murphy, M.M., et al. (2006). Genomic instability and aging-like phenotype in the absence of mammalian SIRT6. Cell 124, 315-329.

Moynihan, K.A., Grimm, A.A., Plueger, M.M., Bernal-Mizrachi, E., Ford, E., Cras-Meneur, C., Permutt, M.A., and Imai, S. (2005). Increased dosage of mammalian Sir2 in pancreatic beta cells enhances glucose-stimulated insulin secretion in mice. Cell Metabol. 2, 105-117.

Nakagawa, T., Lomb, D.J., Haigis, M.C., and Guarente, L. (2009). SIRT5 Deacetylates carbamoyl phosphate synthetase 1 and regulates the urea cycle. Cell 137, 560-570. 
Negrini, S., Gorgoulis, V.G., and Halazonetis, T.D. (2010). Genomic instability--an evolving hallmark of cancer. Nat. Rev. Mol. Cell Biol. 11, 220-228.

North, B.J., and Verdin, E. (2007). Mitotic regulation of SIRT2 by cyclin-dependent kinase 1-dependent phosphorylation. J. Biol. Chem. 282, 19546-19555.

Oberdoerffer, P., Michan, S., McVay, M., Mostoslavsky, R., Vann, J., Park, S.K., Hartlerode, A., Stegmuller, J., Hafner, A., Loerch, P., et al. (2008). SIRT1 redistribution on chromatin promotes genomic stability but alters gene expression during aging. Cell 135, 907-918.

Palacios, J.A., Herranz, D., De Bonis, M.L., Velasco, S., Serrano, M., and Blasco, M.A. (2010). SIRT1 contributes to telomere maintenance and augments global homologous recombination. J. Cell Biol. 191, 1299-1313.

Park, J., Chen, Y., Tishkoff, D.X., Peng, C., Tan, M., Dai, L., Xie, Z., Zhang, Y., Zwaans, B.M., Skinner, M.E., et al. (2013). SIRT5mediated lysine desuccinylation impacts diverse metabolic pathways. Mol. Cell 50, 919-930.

Picard, F., Kurtev, M., Chung, N., Topark-Ngarm, A., Senawong, T., Machado De Oliveira, R., Leid, M., McBurney, M.W., and Guarente, L. (2004). Sirt1 promotes fat mobilization in white adipocytes by repressing PPAR-gamma. Nature 429, 771-776.

Polletta, L., Vernucci, E., Carnevale, I., Arcangeli, T., Rotili, D., Palmerio, S., Steegborn, C., Nowak, T., Schutkowski, M., Pellegrini, L., et al. (2015). SIRT5 regulation of ammoniainduced autophagy and mitophagy. Autophagy 11, 253-270.

Ponugoti, B., Kim, D.H., Xiao, Z., Smith, Z., Miao, J., Zang, M., Wu, S.Y., Chiang, C.M., Veenstra, T.D., and Kemper, J.K. (2010). SIRT1 deacetylates and inhibits SREBP-1C activity in regulation of hepatic lipid metabolism. J. Biol. Chem. 285, 33959-33970.

Qiu, X., Brown, K., Hirschey, M.D., Verdin, E., and Chen, D. (2010). Calorie restriction reduces oxidative stress by SIRT3-mediated SOD2 activation. Cell Metabol. 12, 662-667.

Rodgers, J.T., Lerin, C., Haas, W., Gygi, S.P., Spiegelman, B.M., and Puigserver, P. (2005). Nutrient control of glucose homeostasis through a complex of PGC-1alpha and SIRT1. Nature 434, 113-118.

Ryu, D., Jo, Y.S., Lo Sasso, G., Stein, S., Zhang, H., Perino, A., Lee, J.U., Zeviani, M., Romand, R., Hottiger, M.O., et al. (2014). A SIRT7-dependent acetylation switch of GABPbeta1 controls mitochondrial function. Cell Metabol. 20, 856-869.

Santos, C.R., and Schulze, A. (2012). Lipid metabolism in cancer. FEBS J. 279, 2610-2623.

Sapkota, G.P., Deak, M., Kieloch, A., Morrice, N., Goodarzi, A.A., Smythe, C., Shiloh, Y., Lees-Miller, S.P., and Alessi, D.R. (2002). lonizing radiation induces ataxia telangiectasia mutated kinase (ATM).-mediated phosphorylation of LKB1/STK11 at Thr-366. Biochem. J. 368, 507-516.

Sawada, M., Sun, W., Hayes, P., Leskov, K., Boothman, D.A., and Matsuyama, S. (2003). Ku70 suppresses the apoptotic translocation of Bax to mitochondria. Nat. Cell Biol. 5, 320-329.

Sebastian, C., Zwaans, B.M., Silberman, D.M., Gymrek, M., Goren, A., Zhong, L., Ram, O., Truelove, J., Guimaraes, A.R., Toiber, D. et al. (2012). The histone deacetylase SIRT6 is a tumor suppressor that controls cancer metabolism. Cell 151, 11851199.

Seo, K.S., Park, J.H., Heo, J.Y., Jing, K., Han, J., Min, K.N., Kim, C., Koh, G.Y., Lim, K., Kang, G.Y., et al. (2015). SIRT2 regulates tumour hypoxia response by promoting HIF-1alpha hydroxylation. Oncogene 34, 1354-1362.

Serrano, L., Martinez-Redondo, P., Marazuela-Duque, A., Vazquez, B.N., Dooley, S.J., Voigt, P., Beck, D.B., Kane-Goldsmith, N., Tong, Q., Rabanal, R.M., et al. (2013). The tumor suppressor SirT2 regulates cell cycle progression and genome stability by modulating the mitotic deposition of H4K20 methylation. Genes Dev. 27, 639-653.

Shimazu, T., Hirschey, M.D., Hua, L., Dittenhafer-Reed, K.E., Schwer, B., Lombard, D.B., Li, Y., Bunkenborg, J., Alt, F.W., Denu, J.M., et al. (2010). SIRT3 deacetylates mitochondrial 3hydroxy-3-methylglutaryl CoA synthase 2 and regulates ketone body production. Cell Metabol. 12, 654-661.

Shin, J., He, M., Liu, Y., Paredes, S., Villanova, L., Brown, K., Qiu, X., Nabavi, N., Mohrin, M., Wojnoonski, K., et al. (2013). SIRT7 represses Myc activity to suppress ER stress and prevent fatty liver disease. Cell Rep. 5, 654-665.

Someya, S., Yu, W., Hallows, W.C., Xu, J., Vann, J.M., Leeuwenburgh, C., Tanokura, M., Denu, J.M. and Prolla, T.A. (2010). Sirt3 mediates reduction of oxidative damage and prevention of age-related hearing loss under caloric restriction. Cell 143, 802-812.

$\mathrm{Su}$, T.T. (2006). Cellular responses to DNA damage: one signal, multiple choices. Annu. Rev. Genet. 40, 187-208.

Sundaresan, N.R., Samant, S.A., Pillai, V.B., Rajamohan, S.B., and Gupta, M.P. (2008). SIRT3 is a stress-responsive deacetylase in cardiomyocytes that protects cells from stress-mediated cell death by deacetylation of Ku70. Mol. Cell. Biol. 28, 6384-6401.

Tao, R., Coleman, M.C., Pennington, J.D., Ozden, O., Park, S.H., Jiang, H., Kim, H.S., Flynn, C.R., Hill, S., Hayes McDonald, W., et al. (2010). Sirt3-mediated deacetylation of evolutionarily conserved lysine 122 regulates MnSOD activity in response to stress. Mol. Cell 40, 893-904.

Tennant, D.A., Duran, R.V., and Gottlieb, E. (2010). Targeting metabolic transformation for cancer therapy. Nat. Rev. Cancer $10,267-277$.

Tong, X., Zhao, F., and Thompson, C.B. (2009). The molecular determinants of de novo nucleotide biosynthesis in cancer cells. Curr. Opin. Genet. Dev. 19, 32-37.

Vakhrusheva, O., Braeuer, D., Liu, Z., Braun, T., and Bober, E. (2008a). Sirt7-dependent inhibition of cell growth and proliferation might be instrumental to mediate tissue integrity during aging. J. Physiol. Pharmacol. 59 Supp/ 9, 201-212.

Vakhrusheva, O., Smolka, C., Gajawada, P., Kostin, S., Boettger, T., Kubin, T., Braun, T., and Bober, E. (2008b). Sirt7 increases stress resistance of cardiomyocytes and prevents apoptosis and inflammatory cardiomyopathy in mice. Circ.Res. 102, 703-710.

Vander Heiden, M.G., Cantley, L.C., and Thompson, C.B. (2009). Understanding the Warburg effect: the metabolic requirements of cell proliferation. Science 324, 1029-1033.

Vaquero, A., Scher, M.B., Lee, D.H., Sutton, A., Cheng, H.L., Alt, F.W., Serrano, L., Sternglanz, R., and Reinberg, D. (2006). SirT2 is a histone deacetylase with preference for histone H4 Lys 16 during mitosis. Genes Dev. 20,1256-1261.

Verdin, E., Hirschey, M.D., Finley, L.W., and Haigis, M.C. (2010). Sirtuin regulation of mitochondria: energy production, apoptosis, and signaling. Trends Biochem. Sci. 35, 669-675.

Wang, F., Nguyen, M., Qin, F.X., and Tong, Q. (2007). SIRT2 deacetylates $\mathrm{FOXO} 3 \mathrm{a}$ in response to oxidative stress and caloric restriction. Aging Cell 6, 505-514.

Wang, R.H., Sengupta, K., Li, C., Kim, H.S., Cao, L., Xiao, C., Kim, S., Xu, X., Zheng, Y., Chilton, B., et al. (2008). Impaired DNA damage response, genome instability, and tumorigenesis in SIRT1 mutant mice. Cancer Cell 14, 312-323.

Wang, J.B., Erickson, J.W., Fuji, R., Ramachandran, S., Gao, P., Dinavahi, R., Wilson, K.F., Ambrosio, A.L., Dias, S.M., Dang, C.V., et al. (2010). Targeting mitochondrial glutaminase activity inhibits oncogenic transformation. Cancer Cell 18, 207-219.

Warburg, O. (1956). On the origin of cancer cells. Science 123 , 309-314.

Wise, D.R., and Thompson, C.B. (2010). Glutamine addiction: a new therapeutic target in cancer. Trends Biochem. Sci. 35, 427433.

Yang, C., Sudderth, J., Dang, T., Bachoo, R.M., McDonald, J.G. and DeBerardinis, R.J. (2009). Glioblastoma cells require glutamate dehydrogenase to survive impairments of glucose metabolism or Akt signaling. Cancer Res. 69, 7986-7993.

Yoshizawa, T., Karim, M.F., Sato, Y., Senokuchi, T., Miyata, K., Fukuda, T., Go, C., Tasaki, M., Uchimura, K., Kadomatsu, T., et al. (2014). SIRT7 controls hepatic lipid metabolism by regulating the ubiquitin-proteasome pathway. Cell Metabol. 19, 712-721.

Yuan, H., Wang, Z., Li, L., Zhang, H., Modi, H., Horne, D., Bhatia, R. and Chen, W. (2012). Activation of stress response gene SIRT1 by BCR-ABL promotes leukemogenesis. Blood 119, 1904-1914.

Zhong, L., D'Urso, A., Toiber, D., Sebastian, C., Henry, R.E., Vadysirisack, D.D., Guimaraes, A., Marinelli, B., Wikstrom, J.D., Nir, T., et al. (2010). The histone deacetylase Sirt6 regulates glucose homeostasis via Hif1alpha. Cell 140, 280-293. 